\title{
Estimating Factors Determining Emulsification Capability of Surfactant-Like Peptide with Coarse-Grained Molecular Dynamics Simulation
}

\author{
Tegar Wijaya ${ }^{1}$ and Rukman Hertadi ${ }^{2},{ }^{*}$ \\ ${ }^{1}$ Department of Chemistry, Faculty of Science and Computer, University of Pertamina, \\ Jl. Teuku Nyak Arief, Simprug, Kebayoran Lama, Jakarta 12220, Indonesia \\ ${ }^{2}$ Biochemistry Research Division, Bandung Institute of Technology, Jl. Ganesa No. 10 Bandung 40132, Indonesia
}

* Corresponding author:

tel: +62-22-2502103

email:rukman@chem.itb.ac.id

Received: April 4, 2018

Accepted: September 26, 2018

DOI: $10.22146 /$ ijc. 34547

\begin{abstract}
The ability of surfactant-like peptides to emulsify oil has become the main focus of our current study. We predicted the ability of a series of surfactant-like peptides (G6D, A6D, M6D, F6D, L6D, V6D, and I6D) to emulsify decane molecules using coarsegrained molecular dynamics simulations. A 1- $\mu$ s simulation of each peptide was carried out at $298 \mathrm{~K}$ and $1 \mathrm{~atm}$ using MARTINI force field. Simulation system was constructed to consist of 100 peptide molecules, 20 decane molecules, water, antifreeze particles and neutralizing ions in a random configuration. Out of seven tested peptides, M6D, F6D, L6D, V6D, and I6D were able to form emulsion while G6D and A6D self-assembled to order $\beta$-strands. A higher hydropathy index of amino acids constituting the hydrophobic tail renders the formation of an emulsion by peptides more likely. By calculating contact number between peptides and decanes, we found that emulsion stability and geometry depends on the structure of amino acids constituting the hydrophobic tail. Analysis of simulation trajectory revealed that emulsions are formed by small nucleation following by fusion to form a bigger emulsion. This study reveals the underlying principle at the molecular level of surfactant peptide ability to form an emulsion with hydrophobic molecules.
\end{abstract}

Keywords: coarse-grained molecular dynamic simulation; emulsion; MARTINI; peptide; surfactant

\section{- INTRODUCTION}

A surfactant-like peptide is a group of molecules that consist of amino acids and can decrease interfacial tension. A surfactant-like peptide might solely consist of amino acids, such as V6D2 molecule (Val-Val-Val-ValVal-Val-Asp-Asp), or conjugates of amino acids with lipid. Surfactant-like peptide could be extracted from bacteria such as surfactin which is extracted from Bacillus subtilis [1]. Partial hydrolysis of a protein, such as whey protein, could be used to obtain peptides that could form nanoemulsions [2]. It is also possible to design a surfactant-like peptide by combining polar or charged amino acids as the surfactant head and a series of nonpolar amino acids as the surfactant tails. Such a peptide shows the ability to stabilize membrane protein and to form nanostructures [3-4]. Surfactant-like peptides have some advantages over conventional surfactant such as biocompatibility and rich chemistry that allow modulation of the peptides properties.

To our knowledge, there are at least two applications of surfactant-like peptides: as a building block for nanostructure self-assembly and emulsification agent. Dynamic light scattering and tunneling electron microscopy study show the formation of nanotubes and nanovesicles by a series of peptides GxD2 with varying glycine number [5]. A similar result also obtained using $\mathrm{A} 6 \mathrm{D}$ and $\mathrm{V} 6 \mathrm{D}$ peptides [6]. Some reports reveal the ability of surfactant-like peptides to form an emulsion and stabilize hydrophobic molecule in a polar environment. A series of designer surfactant-like peptides have been used to help stabilize membrane protein during 
purification [3,7]. Lipopeptide from bacteria has been shown to reduce interfacial tension [8].

Furthermore, it also reported that lipopeptide biosurfactant could be produced by bacteria in an oil reservoir [9]. Nutrition supplementation could increase the in-situ production of lipopeptide biosurfactant in oil reservoirs [10]. Due to its compatibility, surfactant-like peptide could be used in food engineering and medical application [11-13].

Despite many reports about the potential of surfactant-like peptides, there is only a little information about surfactant-like peptides in molecular level. Molecular dynamic simulation is powerful tools for investigating molecular properties of surfactant-like peptides. Coarse-grained model, in combination with molecular dynamic simulation, could give insight on the molecular process on hundreds of nanoseconds to microsecond timescale in which self-assembly process more likely to happen. Among many coarse-grained models, the MARTINI model and forcefield are widely used and used in this study [14-15].

In this study, we simulated the emulsification and self-assembly process of a set of surfactant-like peptides namely G6D, A6D, M6D, F6D, L6D, V6D and I6D in the presence of decane molecules. Emulsification or selfassembly process is analyzed based on the difference in the hydrophobic amino acid type of each peptide.

\section{- COMPUTATIONAL METHODS}

\section{Structure Preparation}

The molecular structures of G6D, A6D, M6D, F6D, L6D, V6D, and I6D were constructed using the molefacture plug-in available within Visual Molecular Dynamics (VMD) software [16]. After minimization using CHARMM22 force field in vacuum, the structure was transformed to MARTINI coarse-grained structure using script available at www.cgmartini.nl [17-18].

\section{Simulations details}

Simulation boxes were constructed to consist of 100 peptide molecules, 20 decane molecules, neutralizing ions, antifreeze particles and water. Antifreeze particles were required to tackle the problem with water freezing temperature of the MARTINI model. The peptides and decane molecules were placed randomly inside the box. After minimization of the simulation box, $1-\mu \mathrm{s}$ molecular dynamics simulations were carried out using a MARTINI force-field [14-15]. We used thermal and pressure coupling to maintain the system at a certain temperature $298 \mathrm{~K}$ and $1 \mathrm{~atm}$. Minimization and simulations were performed using the GROMACS molecular package [19-21]. Visualization and the picture used in this report were generated using VMD.

\section{- RESULTS AND DISCUSSION}

Using a coarse-grained model, we have successfully simulated decane emulsification by a series of different peptides with relatively low computational cost. Simulation duration lasted up to $1 \mu$ s and resolved the process of emulsification at the molecular level. The simulation predicts the ability of different peptides to act as a surfactant.

Fig. 1 shows the simulation snapshots at $1 \mu$ s. Out of seven peptides being tested, A6D and G6D form wellordered strands instead of micelle (Fig. 1(a) and 1(b)). These strands consist of peptide molecules antiparallel of each other. This orientation minimizes repulsion force between positive charges of aspartate residue. The simulation snapshots show that the strands do not interact with decane molecules. The other peptides form an emulsion with decane molecules in a micelle-like shape, except for F6D, which formed a tubular emulsion (Fig. 1(c-g)).

The hydrophobic tail of surfactant peptide faces the decane molecules, while the head part (consisting of positively charged aspartate residue) is exposed to water. Most of the F6D, L6D, V6D, and I6D peptide molecules participate in forming emulsion while the number of free peptide molecules is significant in M6D case.

To understand the emulsion formation mechanism, we took a series of simulation snapshots of the I6D system. Fig. 2 shows the snapshots taken at 0 , $12.8,20.1,58.1$, and $97.1 \mathrm{~ns}$. The snapshots suggest that the island mechanism by which small emulsions starts to form is followed by fusion to form bigger emulsions.

We examined the stability and kinetics of emulsion 
(a)

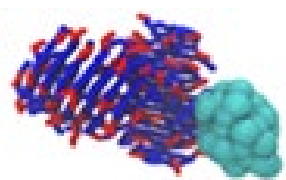

(c)

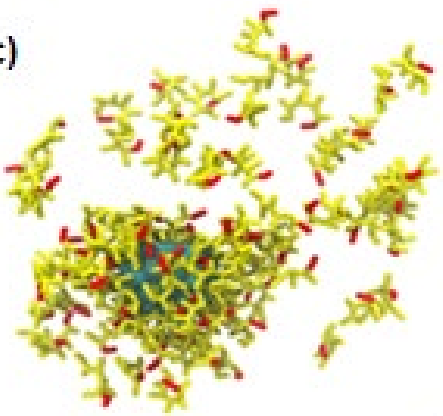

(b)

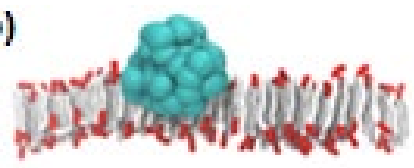

(d)

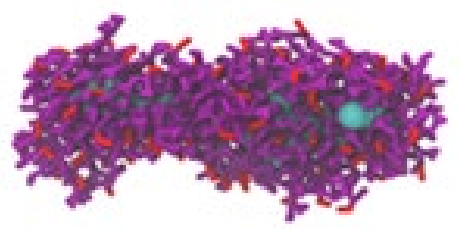

(e)

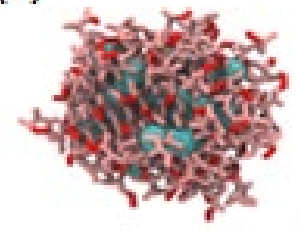

(f)

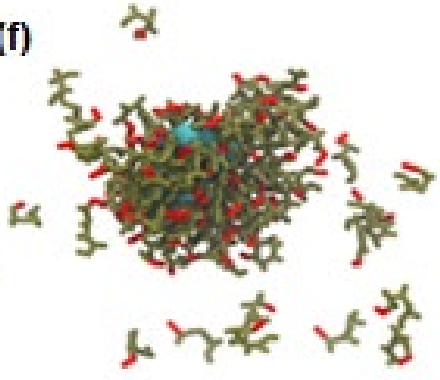

(g)

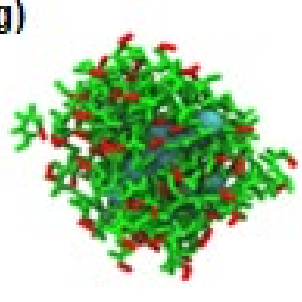

Fig 1. Simulation snapshot at $1 \mu$ s of (a) A6D, (b) G6D, (c) M6D, (d) F6D, (e) L6D, (f) V6D, and (g) I6D. Green balls represent decane molecule while peptides are represented by sticks. Water is not shown for clarity

(a)

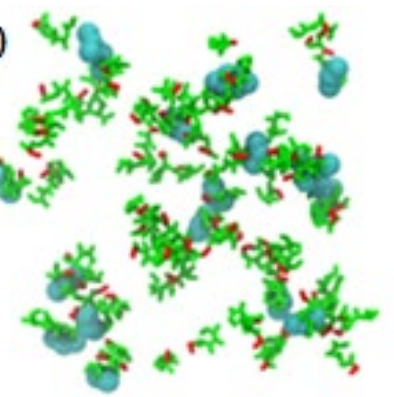

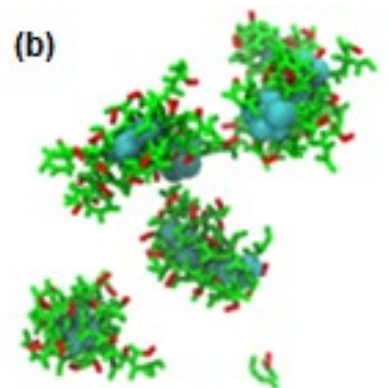

(c)

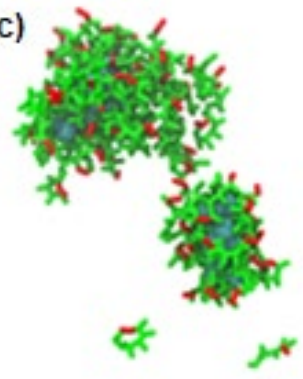

(d)

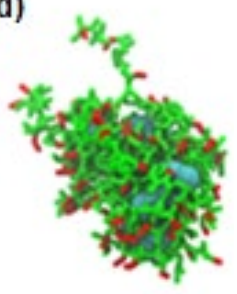

Fig 2. Simulation snapshot of I6D at $0 \mathrm{~ns}$ (a), $12.8 \mathrm{~ns}$ (b), $58.1 \mathrm{~ns}$ (c) and $97.1 \mathrm{~ns}$ (d). Green balls represent decane molecule while peptides are represented by sticks. Water is not shown for clarity

formation by calculating the contact number between decane and peptide molecules. The calculated contact number for a given simulation course is shown in Fig. 3. The trend is similar for all peptides, i.e., a sharp increase shortly after the beginning of the simulation, followed by a stable contact number. In the case of $\mathrm{M} 6 \mathrm{D}$ and V6D, there was a minor decrease before reaching a stable contact number. The stable contact number increased as follows: $\mathrm{M} 6 \mathrm{D}<\mathrm{V} 6 \mathrm{D}<\mathrm{I} 6 \mathrm{D}<\mathrm{L} 6 \mathrm{D}<\mathrm{F} 6 \mathrm{D}$.
The kinetics of micelle formation was investigated by examining the sharp increase of contact number. Fig. 4 shows the contact number up to $50 \mathrm{~ns}$. The graph shows an almost linear increase of contact number in all peptides except for M6D which shows the sigmoid curve. The trend in emulsification speed (gradient of increasing linear part of contact number curve) is the same with the trend of stable contact number.

The simulation results show that the type of residue 


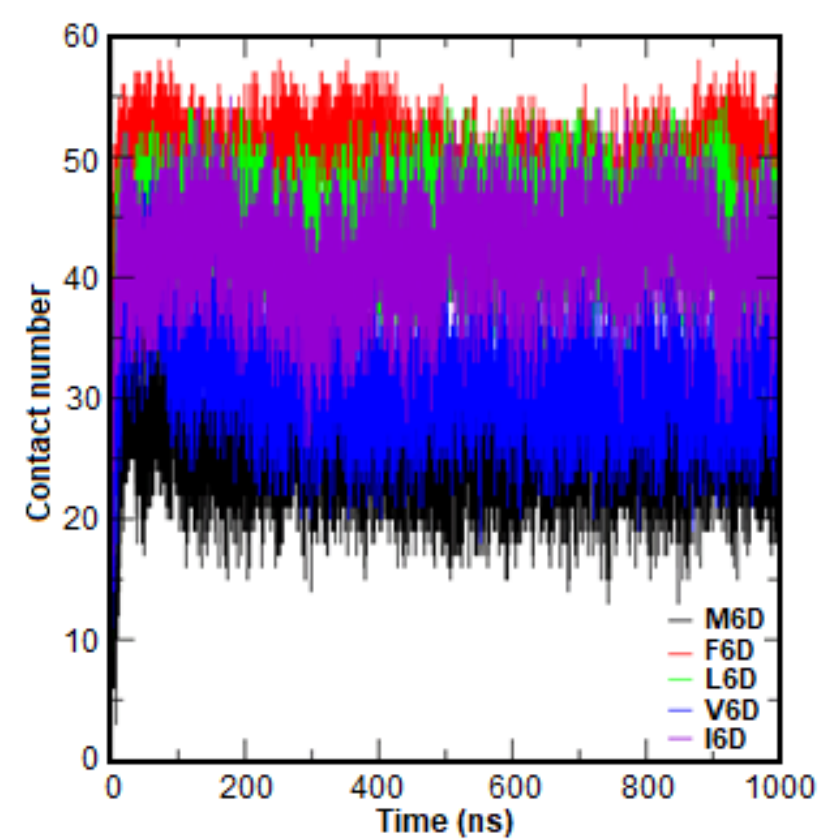

Fig 3. Contact number between peptides and decane molecules during simulations

constituting the hydrophobic tails of surfactant peptide is one of many factors that determine whether the peptide can act as a surfactant. While peptide with alanine and glycine residue could not form an emulsion, peptides with methionine, phenylalanine, leucine, valine, and isoleucine can form an emulsion. We rationalize this observation using the hydropathy index provided by Kyte and Doolittle [22]. Hydropathy index for residue constituting hydrophobic tail of tested peptides are shown in Table 1. The bigger hydropathy index of a residue the bigger its chance to be found buried in a protein, i.e., the more hydrophobic it is. We found that glycine and alanine in G6D and A6D have small hydropathy index the rest of residues being tested here. The simulations suggest that a greater hydropathy index of the residues constituting the hydrophobic tail of the peptide will be associated with a better chance of forming an emulsion. Since the hydropathy index is directly correlated with hydrophobicity, we conclude that hydrophobic interactions are crucial in the emulsification process.

We examined micelle stability by quantifying the contact between peptide and decane molecules during the simulations. Bigger contact number means a stronger hydrophobic interaction that leads to better stability. We

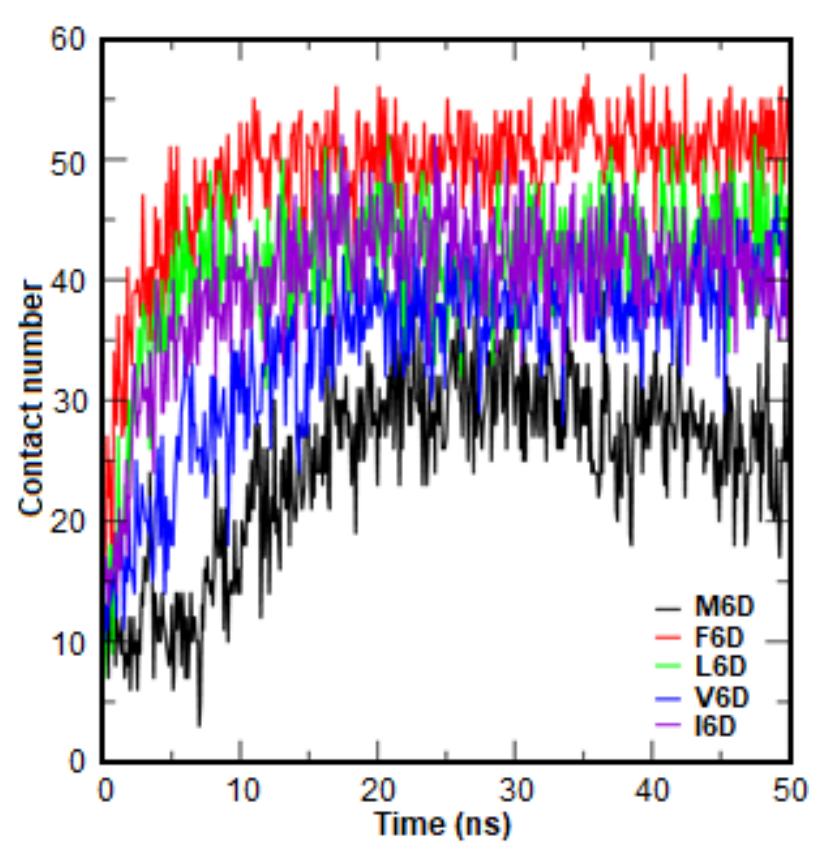

Fig 4. Contact number between peptides and decane molecules near the beginning of simulations

Table 1. Kyte and Doolittle hydropathy index of selected amino acids

\begin{tabular}{lc}
\hline Residue & Hydropathy index \\
\hline Glycine & -0.4 \\
Alanine & 1.8 \\
Methionine & 1.9 \\
Phenylalanine & 2.8 \\
Leucine & 3.8 \\
Valine & 4.2 \\
Isoleucine & 4.5 \\
\hline
\end{tabular}

found that the order of stable contact number, which also means the order of micelle stability does not correlate with the increase in hydropathy index. For example, phenylalanine, despite having relatively low hydropathy index, has the biggest stable contact number. From this observation, we argue that emulsion stability does not depend solely on hydropathy index. Phenylalanine side chain consists of a planar aromatic ring which could provide better contact with decane molecules.

Regarding the process kinetics, we observe that the process is not cooperative for $\mathrm{F} 6 \mathrm{D}, \mathrm{L} 6 \mathrm{D}, \mathrm{I} 6 \mathrm{D}$, and V6D. However, since the curve is sigmoid in the case of M6D, that indicate a possibility of cooperativity. More works 
are needed to discern cooperativity of emulsion formation.

When we examined the snapshots of micelle formation in the I6D system, it was clear that the process started by the nucleation of small micelles was followed by fusion to form bigger micelles. This mechanism could explain the slight decrease in contact before equilibrium is reached in the case of M6D and V6D. The decrease in contact is the result of the fusion of small micelles. After fusion, available surface for contact between decane and peptide molecules decreased accompanied by the release of free peptide. It is indeed that according to Figure 1, the number of free peptide molecules is observed in both the M6D and V6D system than the rest. It suggests that the small micelles do not completely undergo fusion for other system based on the observation that the decane core in the emulsion is less compact than decane aggregate on A6D or G6D. It also further suggests that decane molecules are somewhat dissolved in I6D, L6D, and F6D system.

Despite being unable to form an emulsion with decane, simulations of $\mathrm{A} 6 \mathrm{D}$ and G6D peptides show the interesting result. Both peptides are self-assembled to ordered structure especially in the G6D case in which an ordered strand with the antiparallel orientation of peptides is formed. This is possible due to simplicity in the side chain of alanine and glycine which allow close pack arrangement. In fact, $\mathrm{A} 6 \mathrm{~K}$ peptides, which similar to $\mathrm{A} 6 \mathrm{D}$ peptides, are found to form $\beta$-strands based on FTIR and SSNMR study [23]. The self-assembly process could be controlled to form diverse nanostructures (such as monolayer or cylindrical micelle) by varying $\mathrm{pH}$ or using a surface as a scaffold [24]. The potential for self-assembly of both $\mathrm{A} 6 \mathrm{D}$ and G6D are comparable to phospholipid [25] and lecithin [26] in water that has been demonstrated by using similar computational methods. Therefore, it can be studied further whether both peptides can form liposome-like nanostructure in water as demonstrated computationally for phospholipid [25,27] and lecithin [26].

This study shows that we can use peptide as a surfactant in stabilizing hydrophobic molecule in water. Experimental reports are available where peptides are used to stabilize and help in crystallize membrane-bound protein [3]. Simple surfactant-like peptides have been designed such as A6D, V6D, V6D2, L6D2, A6K, V6K, V6K2 and L6K2 [7].

\section{- CONCLUSION}

Using coarse-grained molecular dynamics simulation, we have successfully described the ability of the surfactant-like peptide to form an emulsion with decane molecules at the molecular level. It was found that the hydropathy index of amino acids constituting the hydrophobic tail is determining the factor of emulsion formation process. The amino acid structure should be taken into consideration when evaluating or predicting emulsion stability. The insights obtained from this study could help in understanding and design peptides to be used as surfactants.

\section{- REFERENCES}

[1] Arima, K., Kakinuma, A., and Tamura, G., 1968, Surfactin, a crystalline peptide-lipid surfactant produced by Isolation, characterization and its inhibition of fibrin clot formation, Biochem. Biophys. Res. Commun., 31 (3), 488-494.

[2] Adjonu, R., Doran, G., Torley, P., and Agboola, S., 2013, Screening of whey protein isolate hydrolysates for their dual functionality: Influence of heat pre-treatment and enzyme specificity, Food Chem., 136 (3-4), 1435-1443.

[3] Zheng, X., Dong, S., Zheng, J., Li, D., Li, F., and Luo, Z., 2014, Expression, stabilization, and purification of membrane proteins via diverse protein synthesis systems and detergents involving cell-free associated with self-assembly peptide surfactants, Biotechnol. Adv., 32 (3), 564-574.

[4] Hamley, I.W., 2014, Peptide Nanotubes, Angew. Chem. Int. Ed., 53 (27), 6866-6881.

[5] Santoso, S., Hwang, W., Hartman, H., and Zhang, S., 2002, Self-assembly of surfactant-like peptides with variable glycine tails to form nanotubes and nanovesicles, Nano Lett., 2 (7), 687-691.

[6] Vauthey, S., Santoso, S., Gong, H., Watson, N., and Zhang, S., 2002, Molecular self-assembly of surfactant-like peptides to form nanotubes and 
nanovesicles, Proc. Natl. Acad. Sci. U.S.A., 99 (8) 5355-5360.

[7] Zhao, X., 2009, Design of self-assembling surfactantlike peptides and their applications, Curr. Opin. Colloid In., 14 (5) 340-348.

[8] Youssef, N., Simpson, D.R., Duncan, K.E., McInerney, M.J., Folmsbee, M., Fincher, T., and Knapp, R.M., 2007, In situ biosurfactant production by Bacillus strains injected into a limestone petroleum reservoir, Appl. Environ. Microbiol., 73 (4), 1239-1247.

[9] Youssef, N.H., Nguyen, T., Sabatini, D.A., and McInerney, M.J., 2007, Basis for formulating biosurfactant mixtures to achieve ultra-low interfacial tension values against hydrocarbons, $J$. Ind. Microbiol. Biotechnol., 34 (7), 497-507.

[10] Simpson, D.R., Natraj, N.R., McInerney, M.J., and Duncan, K.E., 2011, Biosurfactant-producing Bacillus are present in produced brines from Oklahoma oil reservoirs with a wide range of salinities, Appl. Microbiol. Biotechnol., 91 (4), 1083-1093.

[11] Adjonu, R., Doran, G., Torley, P., and Agboola, S., 2014, Whey protein peptides as components of nanoemulsions: A review of emulsifying and biological functionalities, J. Food Eng., 122 (1), 15-27.

[12] Singh, P., and Cameotra, S.S., 2004, Potential applications of microbial surfactants in biomedical sciences, Trends Biotechnol., 22 (3), 142-146.

[13] Panda, J.J., and Chauhan, V.S., 2014, Short peptidebased self-assembled nanostructures: Implications in drug delivery and tissue engineering, Polym. Chem., 5 (15), 4418-4436.

[14] Marrink, S.J., Risselada, H.J., Yefimov, S., Tieleman, D.P., and de Vries, A.H., 2007, The MARTINI force field: A coarse-grained model for biomolecular simulations, J. Phys. Chem. B, 111 (27), 7812-7824.

[15] Monticelli, L., Kandasamy, S.K., Periole, X., Larson, R.G., Tieleman, D.P., and Marrink, S.J., The MARTINI coarse-grained force field: Extension to proteins, J. Chem. Theory Comput., 4 (5), 819-834.

[16] Humphrey, W., Dalke, A., and Schulten, K., 1996, VMD: Visual molecular dynamics, J. Mol. Graphics., 14 (1), 33-38.
[17] MacKerell, A.D., Bashford, D., Bellot, M., Dunbrack, R.L., Evanseck, J.D., Field, M.J., Fischer, S., Gao, J., Guo, H., Ha, S., Joseph-McCarthy, D., Kuchnir, L., Kuczera, K., Lau, F.T.K., Mattos, C., Michnick, S., Ngo, T., Nguyen, D.T., Prodhom, B., Reiher, W.E., Roux, B., Schlenkrich, M., Smith, J.C., Stote, R., Straub, J., Watanabe, M., WirkiewiczKuczera, J., Yin, D., and Karplus, M., 1998, Allatom empirical potential for molecular modeling and dynamics studies of proteins, J. Phys. Chem. B, 102 (18), 3586-3616.

[18] MacKerell, A.D., Feig, M., and Brooks, C.L., 2004, Extending the treatment of backbone energetics in protein force fields: Limitations of gas-phase quantum mechanics in reproducing protein conformational distributions in molecular dynamics simulations, J. Comput. Chem., 25 (11), 1400-1415.

[19] Lindahl, E., Hess, B., and van der Spoel, D., 2001, GROMACS 3.0: A package for molecular simulation and trajectory analysis, J. Mol. Model., 7 (8), 306-317.

[20] Berendsen, H.J.C., van der Spoel, D., and van Drunen, R., 1995, GROMACS: A message-passing parallel molecular dynamics implementation, Comput. Phys. Commun., 91 (1-3), 43-56.

[21] van der Spoel, D., Lindahl, E., Hess, B., Groenhof, G., Mark, A.E., Berendsen, H.J., 2005, GROMACS: fast, flexible, and free, J. Comput. Chem., 26 (16), 1701-1718.

[22] Kyte, J., and Doolittle, R.F., 1982, A simple method for displaying the hydropathic character of a protein, J. Mol. Biol., 157 (1), 105-132.

[23] Middleton, D.A., Madine, J., Castelletto, V., and Hamley, I.W., 2013, Insights into the molecular architecture of a peptide nanotube using FTIR and solid-state NMR spectroscopic measurements on an aligned sample, Angew. Chem. Int. Ed., 52 (40), 10537-10540.

[24] Qiu, F., Chen, Y., and Zhao, X., 2009, Comparative studies on the self-assembling behaviors of cationic and catanionic surfactant-like peptides, J. Colloid Interface Sci., 336 (2), 477-484. 
[25] Hudiyanti, D., Radifar, M., Raharjo, T.J., Narsito, N., and Noegrohati, S., 2014, A Coarse-grained molecular dynamics simulation using NAMD package to reveal aggregation profile of phospholipid self-assembly in water, J. Chem., 2014, 273084.

[26] Dwiastuti, R., Radifar, M., Marchaban, M., Noegrohati, S., and Istyastono, E.P., 2016, Molecular dynamics simulations and empirical observations on soy lecithin liposome preparation, Indones. J. Chem., 16 (2), 222-228.

[27] Koshiyama, K., and Wada, S., 2016, Collapse of a lipid-coated nanobubble and subsequent liposome formation, Sci. Rep., 6, 28164. 